\section{¿Es tiempo de modificar la vinculación pregrado postgrado en la educación médica chilena? Reflexiones sobre una vía de doble titulación Médico cirujano/Doctor en ciencias médicas}

\author{
MIGUEL CONCHA M. ${ }^{\mathrm{a}}$
}

\section{Time to change the graduate-postgraduate liaison in Chilean medical education? Reflections about a double certification as Physician and Doctor in medical sciences}

In the last 150 years, scientific research has produced extraordinary discoveries in Medicine and there is no doubt that research will continue contributing substantially in the future but there is no the same conviction regarding how to provision such capacities in medical graduates. In Chile, the Faculties of Medicine created several doctorate programs in Medical Sciences (Ph.D.) to strengthen medical research. However, the low number of physicians who apply to these programs is a caveat. These programs provide the advanced third-cycle competencies expected by students aspiring for an excellence research certification and their incorporation into academia. Universities stand out in their capacity to adapt themselves to educational needs, developing programs designed to fill specific gaps. Ph.D. programs intercalated to the medical career show that this is the correct approach. The development of specific doctorate programs for the small number of physicians interested in research and for medical students with a strong interest in research could be an innovative solution to motivate and encourage them to develop a career in clinical investigation. Using this approach, Medical Schools and Doctorate in Medical Sciences programs would jointly stimulate the training of medical scientists.

(Rev Med Chile 2017; 145: 1569-1578)

Key words: Medical education, research career, physician-scientist, MD/ PhD program, Chile.
Director Escuela de Graduados de Medicina (2009-2017), Facultad de Medicina, Universidad Austral de Chile. Valdivia, Chile.

${ }^{a}$ Doctor en Ciencias.

Declaración de Potenciales Conflictos de Interés: El autor declara que no existe un posible conflicto de intereses en el presente manuscrito.

El trabajo no recibió financiamiento.

Recibido el 28 de junio de 2017, aceptado el 18 de diciembre de 2017.

Correspondencia a: Dr. Miguel Concha Instituto de Anatomía, Histología y Patología, Facultad de Medicina, UACh. Cas. 567, Valdivia, Chile. Teléfono -(56)-63-2-293013 migconch@uach.cl
L a Comisión Nacional de Acreditación (CNA-Chile) en su Resolución N629 de Acreditación del Doctorado en Ciencias Médicas, Universidad Austral de Chile (UACh) señaló que no se observaban avances significativos en el ingreso de estudiantes médicos cirujanos y que sería conveniente explorar modelos internacionales que aborden el área de la investigación clínica a nivel de postgrado ${ }^{1}$.

El Doctorado en Ciencias Médicas UACh fue creado el año 2010 con el objeto de formar "doctores con competencias del más alto nivel en 
investigación biomédica con proyección clínica, en las diferentes disciplinas del área de la salud, a fin de fortalecer la capacidad de investigación $y$ transferencia tecnológica del pais"'. Inicialmente fue ofertado de preferencia a postulantes médicos cirujanos. La entrega del grado, al igual que los Doctorados en Ciencias existentes en el país, requiere la aprobación de asignaturas, del examen de calificación o proyecto de tesis, una investigación original, su defensa y disertación solemne. Con la creación del Programa se asumió el anhelo de que el ingreso de nuevos académicos con grado de doctor, con habilidades para realizar investigación avanzada, potenciarían el área clínica de la Facultad de Medicina. Sin embargo, la constatación de la reducida matrícula de médicos cirujanos en uno de los programas que ya existían en el país, condujo a poner una cuota de cautela respecto a las efectivas posibilidades de formar médicos cirujanos doctores en ciencias, aclarándose en los objetivos que "igualmente el programa abre sus puertas a profesionales de la salud y en general del área biomédica que muestren un marcado interés por la investigación con proyección clínica"2.
De los tres alumnos médicos cirujanos matriculados el 2011 uno de ellos renunció al año siguiente y de dos nuevos ingresos ocurridos posteriormente uno también renunció. Al 2017 los médicos cirujanos matriculados representaban $32 \%$ de los alumnos; si se restan las deserciones y la matrícula de médicos extranjeros, la cifra disminuye a $12 \%$. Etas cifras no son diferentes a los programas de doctorado ofrecidos por las Facultades de Medicina chilenas (Tabla 1) que declaran la intención por el ingreso de médicos cirujanos (Tabla 2).

Anteriormente Salas y Rigotti habían mostrado que el número de proyectos FONDECYT del área de Medicina liderados por médicos habían disminuido desde $80 \%$ en el año 1984 a 50\% el año $2003^{3}$. Un estudio posterior de los programas de médico especialista que se dictan en Chile reveló el vacío existente entre la intensión declarada por la investigación y la efectiva productividad científica de profesores y residentes ${ }^{4}$. En Brasil, en 1985, 52\% de los profesores de los institutos médicos de la Universidad Federal de Río Janeiro eran médicos. Su número cayó a 25\% en 1990 y más recientemente se calculó en $5 \%{ }^{5}$. En Estados

Tabla 1. Programas de Doctorado ofrecidos por las Facultades de Medicina Chilenas

\begin{tabular}{|c|c|c|c|}
\hline Universidades $^{1}$ & $\begin{array}{l}\text { Doctorados en ciencias } \\
\text { médicas }\end{array}$ & Otros doctorados & $\begin{array}{l}\text { Estado } \\
\text { acreditación }\end{array}$ \\
\hline de Valparaíso & & $\begin{array}{l}\text { Estudios Interdisciplinarios sobre } \\
\text { Pensamiento, Cultura y Sociedad }\end{array}$ & Acreditado \\
\hline \multirow[t]{5}{*}{ de Chile } & Ciencias Médicas y Especialidad & & Acreditado \\
\hline & & Ciencias Biomédicas & Acreditado \\
\hline & & Nutrición y Alimentos & Acreditado \\
\hline & & Psicoterapia ${ }^{3}$ & Acreditado \\
\hline & & Salud Pública & Acreditado \\
\hline \multirow[t]{2}{*}{ P. Univ. Católica de Chile } & Ciencias Médicas & & Acreditado \\
\hline & & Neurociencias & Acreditado \\
\hline del Desarrollo & Ciencias Médicas & & No acreditado \\
\hline de Los Andes & BioMedicina & & No acreditado \\
\hline Concepción & & Salud Mental & Acreditado \\
\hline \multirow[t]{2}{*}{ de la Frontera } & Ciencias Médicas & & Acreditado \\
\hline & & Ciencias Morfológicas & Acreditado \\
\hline Austral de Chile & Ciencias Médicas & & Acreditado \\
\hline
\end{tabular}

${ }^{1}$ Los programas fueron identificados mediante revisión de las páginas web de las universidades chilenas que ofrecen la carrera de Medicina. La acreditación de los programas fue corroborada por consulta en https://www.cnachile.cl/Paginas/buscador-avanzado.aspx. ${ }^{2}$ Corresponde a programa de doctorado interfacultades. ${ }^{3}$ Programa de doctorado interuniversitario. 
Tabla 2. Matricula de Médicos Cirujanos en Programas de Doctorado ofrecidos por Facultades de Medicina en Chile (2011-2016)

\begin{tabular}{|cccccccc|}
\hline Programas $^{\mathbf{1}}$ & $\mathbf{2 0 1 1}$ & $\mathbf{2 0 1 2}$ & $\mathbf{2 0 1 3}$ & $\mathbf{2 0 1 4}$ & $\mathbf{2 0 1 5}$ & $\mathbf{2 0 1 6}$ & Total $^{\mathbf{2}}$ \\
\hline $1^{3}$ & $4 / 4$ & $1 / 1$ & $2 / 2$ & $3 / 3$ & $4 / 4$ & $4 / 4$ & $18 / 18$ \\
& $(100 \%)$ & $(100 \%)$ & $(100 \%)$ & $(100 \%)$ & $(100 \%)$ & $(100 \%)$ & $(100 \%)$ \\
24 & $2 / 7$ & $3 / 7$ & $0 / 5$ & $2 / 6$ & $2 / 7$ & $1 / 8$ & $10 / 40$ \\
& $(28,57 \%)$ & $(42,85 \%)$ & $(0 \%)$ & $(33,33 \%)$ & $(28,57 \%)$ & $(12,5 \%)$ & $(25 \%)$ \\
3 & $0 / 3$ & $0 / 6$ & $0 / 4$ & $0 / 4$ & $0 / 7$ & $3 / 7$ & $3 / 31$ \\
& $(0 \%)$ & $(0 \%)$ & $(0 \%)$ & $(0 \%)$ & $(0 \%)$ & $(42,85 \%)$ & $(9,67 \%)$ \\
4 & - & - & - & $1 / 5$ & $1 / 1$ & $0 / 4$ & $2 / 10$ \\
& & & & $(20 \%)$ & $(100 \%)$ & $(0 \%)$ & $(20 \%)$ \\
5 & $0 / 4$ & $2 / 3$ & $0 / 1$ & $0 / 9$ & $0 / 5$ & $0 / 3$ & $2 / 25$ \\
& $(0 \%)$ & $(66,66 \%)$ & $(0 \%)$ & $(0 \%)$ & $(0 \%)$ & $(0 \%)$ & $(8 \%)$ \\
& $1 / 4$ & $2 / 4$ & $0 / 4$ & $0 / 4$ & $1 / 4$ & $1 / 5$ & $5 / 25$ \\
& $(25 \%)$ & $(50 \%)$ & $(0 \%)$ & $(0 \%)$ & $(25 \%)$ & $(20 \%)$ & $(20 \%)$ \\
& $3 / 5$ & $0 / 2$ & $1 / 2$ & $0 / 4$ & $1 / 3$ & $0 / 4$ & $5 / 20$ \\
& $(60 \%)$ & $(0 \%)$ & $(50 \%)$ & $(0 \%)$ & $(33,33 \%)$ & $(0 \%)$ & $(25 \%)$ \\
& $10 / 27$ & $8 / 23$ & $3 / 18$ & $6 / 35$ & $9 / 31$ & $9 / 35$ & $45 / 169$ \\
& $(37,03 \%)$ & $(34 \%)$ & $(16,66)$ & $(17,14 \%)$ & $(24,32 \%)$ & $(25,71 \%)$ & $(26,62 \%)$ \\
\hline
\end{tabular}

'De los 14 programas de Doctorado listados en la Tabla 1 se incluyeron solo aquellos que declararon un interés neto por el ingreso de médicos cirujanos. Todos ellos menos uno corresponden a Doctorados en Ciencias Médicas o Biomedicina. El número de matriculados se obtuvo mediante consentimiento informado y acuerdo de reserva de confidencialidad. ${ }^{2}$ Total de médicos cirujanos matriculados vs total de matrículas en el período. ${ }^{3} \mathrm{El}$ programa declara como objetivo formar un Médico Especialista Doctor en Ciencias Médicas por lo que se ofrece exclusivamente para médicos cirujanos. El ingreso de médicos cirujanos es semejante al de algunos programas MD/PhD pero el número de matriculados resulta moderado si se considera que la misma Facultad de Medicina incorpora cada año aproximadamente 430 médicos en programas de médico especialista. ${ }^{4} \mathrm{El}$ doctorado está orientado a un área específica de la Medicina y declara su interés por el ingreso de médico cirujanos.

Unidos de América (EUA) los médicos captaban en $1967,43 \%$ de los proyectos de investigación otorgados por los National Institutes of Health (NIH) y en 1999 solo $25 \% 6$.

Así, no resultó extraño que Wyngaarden anticipara que los médicos científicos eran "una especie en peligro" . Entre los factores concurrentes se han mencionado la creciente participación en temas de Medicina de investigadores no médicos con contratos universitarios a tiempo completo; la presión social; la brecha existente entre las remuneraciones universitarias y las pagadas por los prestadores clínicos públicos y privados; y la demandante dedicación a los estudios exigida a los estudiantes de Medicina ${ }^{8}$. No menor, en Chile las políticas de formación de médicos especialistas del último decenio han alcanzado niveles de inversión estatal que duplican el gasto nacional en formación de Doctores en Ciencias.
En este escenario, la observación realizada por la CNA-Chile es pertinente y atendible.

\section{El grado de doctor en filosofía}

Park ha apuntado que a menudo en las ceremonias universitarias se escucha mencionar que los doctorados son "el pináculo del éxito académico", "el zenit del aprendizaje" o "la cumbre de las becas universitarias" pero que no cabe duda que es el grado académico más alto con que una universidad reconoce a un estudiante que ha completado un programa de trabajo en un área particular del conocimiento 9 . Es el grado de investigación por excelencia.

Los actuales programas se remontan a la simbiosis producida entre idealismo y política que dio como fruto la Universidad de Berlín en octubre 
de $1810^{10,11}$. Su creación interrumpe el aciago camino que seguían las universidades europeas hasta entonces. Si al rol de la nueva universidad, sus creadores, W. Humboldt, F. Schleiermacher, J. Fichte, C. Wolf, F. Schelling, entre otros, lograron sumar la libre creación científica a la enseñanza que le interesaba al Estado (Medicina, Derecho y Teología), también consiguieron engarzar la filosofía como su centro natural ${ }^{12}$. Humboldt dejó nítidamente expresado en el proyecto de creación de la universidad que ésta no se podía limitar al rol de trasmisora del saber puesto que, desde el punto de vista de la investigación, el saber jamás se alcanza si no que resta como una pregunta abierta sin límites ${ }^{12}$. Agregó que el profesor universitario no es más un profesor y el estudiante no es alguien meramente enganchado en el proceso de aprendizaje si no una persona que asume su propia investigación, mientras el profesor lo dirige y apoya ${ }^{13}$. En cuánto a la filosofía, "la primera y soberana", Schleiermacher y Shelling sostuvieron que era la más apropiada para liberar al espíritu de la estrechez de una formación unilateral y de elevarlo hasta el reino de lo universal y de lo absoluto, a la que "todos los miembros de la universidad, sea cuál sea la Facultad a la que pertenecieran, le debían sus raíces"12. La libertad para investigar y la unidad entre investigación y enseñanza quedaron así ancladas institucionalmente, bajo el amparo de la filosofía.

Al considerar el doctorado como un grado de investigación, la Universidad de Berlín dio origen a su redefinición y subsecuente difusión univer$\mathrm{sal}^{14}$, el cual con las denominaciones de $P h D$, DPhil, D.Lit, D.Sc, LL.D o Doctorado es ofrecido actualmente por todas las disciplinas académicas, incluida la Medicina.

\section{Un efecto de Bologna sobre la carrera de medicina}

Los extraordinarios cambios en la educación superior inspirados en las declaraciones de la Sorbonne y luego de Bologna han derivado en un intenso trabajo de acuerdos e implementación por parte de Organismos Internacionales, Estados, universidades, carreras y programas. Implementadas bajo una estructura de tres ciclos formativos (bachelor, master y doctorado), las innovaciones se han movido desde las estructurales, como la creación del Espacio Europeo de Educación Superior, a otras de gestión académica tales como el establecimiento de sistemas de aseguramiento de la calidad, marcos de comparación de carreras y grados, créditos transferibles, y movilidad de estudiantes y profesores ${ }^{15,16}$. Latinoamérica adhirió a esta tarea en sintonía con la aspiración de un cambio global en la educación superior ${ }^{17}$.

La adopción del sistema de tres ciclos resultó particularmente controversial en Medicina; no obstante, el reconocimiento del bachelor (para los primeros años de estudios) y master (al egreso)ha alcanzado aceptación general ${ }^{18,19}$. Así, Bélgica, Holanda, Dinamarca, Suiza, Portugal y otros países la habían adoptado al año 2014 ${ }^{19-21}$; también Francia bajo la denominación de diplômes ${ }^{22}$. Otros, como Alemania, acogieron la estructura del bachelor para los primeros tres años ${ }^{23}$; España, eliminó la licenciatura por el grado y concede el máster a la finalización de los estudios ${ }^{24,25}$; Irlanda y el Reino Unido, aunque conservan como calificación primaria el Bachelor of Medicine y Bachelor of Surgery/Chirurgery (Universidad de Cambridge: $M B$ $B C h i r)$ éste es reconocido como cualificación de segundo ciclo $^{26}$. Más allá del ámbito Europeo, en la mayoría de los países anglosajones la carrera de Medicina fue históricamente homologada al grado de doctor (Doctor in Medicine [MD], Doctor in Osteopatic Medicine [DO]), pudiendo incluirse en este grupo el grado alemán de Doctor en Medicina (Dr. med.) que es otorgado luego de terminados los estudios de pregrado, pero solo una vez aprobado el Staatsprüfung y la disertación. No obstante, los grados de $M D$ y $D O$ no son reconocidos como doctorados de investigación por el National Council for Educational Statistics y la National Science Foundation $^{27}$, y existe una recurrente discusión acerca de la calidad del grado Dr. med $^{28}$. Suiza actuó aún más radicalmente al reemplazar con el Master en Medicina el grado de Dr. med. que se entregaba al finalizar los seis años de estudios ${ }^{21,29}$.

Aunque el alineamiento progresivo de los países con el Framework for Qualifications of the European Higher Education Area (QF-EHEA) ha permitido que prevalezca la visión de que el grado garantizado para los estudios de Medicina es de segundo ciclo (master), las competencias académicas y/o profesionales esperadas para este nivel [Nivel CINE 7, UNESCO] ${ }^{30}$ no otorgan la condición de independencia deseable en los estudiantes que aspiran a una certificación de investigación 
avanzada como el grado de doctor (Nivel CINE 8). En consecuencia, a pesar de las innovaciones efectuadas, los modelos internacionales que abordan la formación de médicos investigadores deben ser indagados en otros ámbitos.

\section{Los programas de doble titulación $\mathrm{MD} / \mathrm{PhD}$}

La investigación ha sido la fuerza que ha conducido los asombrosos descubrimientos en Medicina de los últimos 150 años y no cabe duda que continuará haciéndolo en el futuro. Difícilmente esta aseveración despertaría controversia en la comunidad académica o el público en general. Sin embargo, no existe la misma convicción sobre cómo provisionar tales capacidades en los egresados. Las buenas universidades se han distinguidos por adaptarse a las demandas educacionales desarrollando programas diseñados de acuerdo a nichos específicos. Esta aproximación, basada en la diversificación y diferenciación, ha probado ser exitosa9.

Los programas de doble graduación Médico Cirujano y Doctor en Filosofía $(M D / P h D)$ han sido considerados como uno de los sistemas de entrenamiento más útiles para realizar una carrera en medicina orientada a la investigación ${ }^{31}$. La idea se originó en la Escuela de Medicina de la Case Western Reserve University (Ohio), luego se expandió a otros estados y más tarde a universidades europeas y de los países del Asia Pacífico. Las Universidades de Harvard, Cambridge y Heidelberg, entre muchas otras, ofrecen cada año a un número reducido y selecto de alumnos de la carrera de Medicina estudios equivalentes a un $P h D^{32-35}$. La formación se efectúa intercalada durante los estudios de Medicina y el estudiante recibe al egresar tanto el título (o grado según el caso) de médico cirujano y el grado de $P h D$. De acuerdo al plan de estudios de Cambridge, el ingreso al programa $M B / P h D$ ocurre al $4^{\circ}$ año luego de haber aprobado con alto puntaje el examen FINAL MB Part I (Figura 1). A continuación se efectúan aproximadamente cuatro años de doctorado para volver al quinto año a terminar la carrera de Medicina. El grado de $P h D$ se entrega junto con el grado de $M B$ al egreso de los alumnos luego de aprobar el sexto año ${ }^{34}$.

Kuehnle y col han recordado el notable concepto en que se basan los programas $M D / P h D$ apuntando que "el entrenamiento de investigador nutre el conocimiento médico y el cuidado del paciente $y$, simultáneamente, el entrenamiento médico claramente influencia el foco y la práctica de la investigación clínica" ${ }^{\prime 3}$. Bonham ha destacado que los programas de titulación combinada entregan entrenamiento integrado y riguroso y que pocos se atreverían a cuestionar la influencia que han tenido en el progreso de las Ciencias ${ }^{37}$. Observó que más de la mitad de quienes recibieron el premio Nobel en Fisiología o Medicina entre 1997 y el 2013 egresaron de estos programas o contaban con uno de estos médicos en sus equipos de trabajo.

Mediante las becas de formación Medical Scientist Training Program los NHI han apoyado desde 1970 los programas de más o menos 40 Escuelas de Medicina ${ }^{38}$. Otras 75 Escuelas de Medicina (EUA) ofrecen el programa con sus propios recursos. Entre 1995 y el 2000 un total de 2.580 estudiantes recibieron becas de estudio y al 2011 el aporte presupuestario alcanzaba a US \$94 millones sin considerar la contribución de las Escuelas de Medicina ${ }^{38}$. Es muy sugerente el elevado porcentaje de los egresados que se dedican a la investigación y la carrera académica y el reducido número de ellos que deriva a la práctica privada como revelan la Universidad de Pensilvania, el programa interinstitucional Weill Cornell/ Rockefeller/Sloan Katering o los datos nacionales de EUA $^{39-41}$.

A propósito del programa del University College of London, Stewart comentó que los $M B /$ $P h D$ no reemplazan a un $P h D$ si no que permiten que aquellos jóvenes fuertemente motivados por la investigación puedan a una edad más temprana realizar un $P h D^{42}$. El ingreso anual a este programa varía entre 2 y 6 alumnos y los matriculados hasta abril de 2012 habían publicado 622 artículos sin considerar libros o proceeedings de conferencias.

La Universidad de Heidelberg ha justificado la disponibilidad de un programa $M D / P h D$ compartido entre las Facultades de Medicina y de Ciencias Naturales y Matemáticas en la confianza que estudiantes especialmente talentosos de $\mathrm{Me}$ dicina y Odontología, interesados en la Ciencia y que han aprobado el primer sistema de exámenes de la carrera, se preparen como investigadores a fin de estudiar las causas y progresión de las enfermedades, su prevención, reconocimiento y tratamiento ${ }^{35}$. El programa admite solo 6 estudiantes por año. 


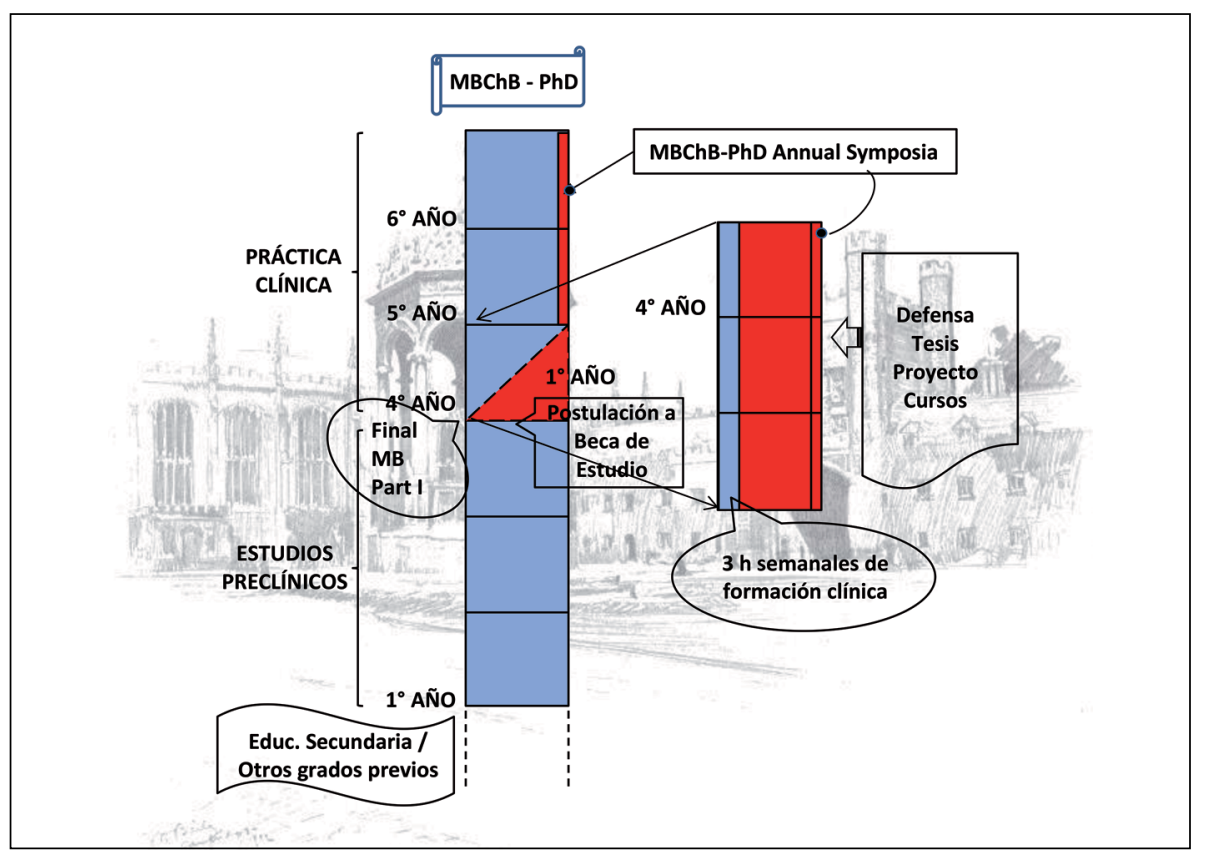

Figura 1. Programa MBChB/PhD Universidad de Cambridge. El ingreso ocurre al $4^{\circ}$ año de la carrera de Medicina luego de haber sido aprobado con alto puntaje el examen FINAL MB Part I. El $1^{\circ}$ año del programa es mixto porque el estudiante efectúa un curso clínico principal, el Core Clinical Practice, pero además un módulo de Academic Medicine, investiga opciones de proyecto de Tesis, se le ha asignado un supervisor y se habrá adjudicado una beca de estudios. Luego siguen tres años con dedicación preferente a la investigación, en que además efectúa algunos cursos que le entregan competencias para desarrollar la Tesis y desempeñarse como investigador. En este período dedica tres horas a la semana a actividades clínicas. Al termino el estudiante vuelve a la clínica donde efectúa la Práctica Clínica Especializada ( $5^{\circ}$ año) y luego la Práctica Clínica Aplicada ( $6^{\circ}$ año), quedando habilitado para recibir la doble titulación de $M B C h S / P h D$. Durante los años en el programa $M B C h B / P h D$ asiste regularmente al Simposio Anual $M B / P h D$. La estructura de los programas $M D / P h D$ es más o menos semejante al aquí presentado. La vía convencional de los estudios de Medicina se muestra en azul; el programa MB/PhD, en rojo. [En el dibujo Trinity College, University of Cambridge, por Vernon Howe Bailey (1902). University of Toronto and Internet Archive, http:// www.victorianweb.org/victorian/painting/bailey/16.html].

Hasta donde conocemos, ninguna universidad iberoamericana desarrolla este modelo con excepción de la Universidad Federal de Río Janeiro. Según lo informado por Oliveira y col su creación respondió a una estrategia para mejorar y expandir la investigación biomédica en Brasil ${ }^{5,8}$. Oliveira subraya que la iniciativa ha resultado exitosa y que fue posible organizar el programa no obstante la rígida legislación universitaria del país y la resistencia de grupos académicos conservadores. Los primeros 6 médicos que ingresaron al programa piloto inicial han publicado hasta la fecha un total de 223 artículos científicos. Dos de ellos son profesores universitarios y dos trabajan en institutos científicos, los otros dos se dedican al ejercicio privado de la Medicina. El programa ha logrado cumplir sus objetivos respecto a productividad científica, visibilidad nacional e internacional, y estímulo a la carrera académica y científica de los médicos pero enfrenta el desafío de elevar el impacto internacional de las publicaciones, aumentar el interés motivacional de los estudiantes que rutinariamente están sobrepasados por los estudios clínicos, y procurar incentivos económicos de organismos del Estado para los alumnos interesados.

\section{Perspectivas}

El año 2010 "The Commission on Education of Health Professionals for the $21^{\text {st }}$ Century" recomendó un nuevo modelo de educación médica que enfatiza la flexibilidad y la adaptación de los cu- 
rrículos a las necesidades locales ${ }^{43}$. A pesar de este avance, ciertos aspectos de la educación médica sobrellevan persistentes dificultades, entre ellos la participación de los estudiantes en investigación. La declinación del número de investigadores médicos se ha hecho ostensible a nivel global.

A fin de enfrentar este problema las escuelas de Medicina han formulado diversas opciones como los programas "honor" de investigación, proyectos de investigación continua o discontinua durante la carrera y programas de carácter obligatorio, todos los cuales tienden a fortalecer currículos, aumentar las posibilidades de ingreso a programas de médico especialista e inspirar también el interés por la investigación ${ }^{44}$. No obstante que la participación de los estudiantes en investigación es altamente recomendable, ésta no es suficiente y se requiere avanzar en la formación mediante el ingreso de un número selecto de estudiantes a programas de investigación avanzados.

En Chile, las Facultades de Medicina han creado Doctorados en Ciencias Médicas en los cuales habitualmente los médicos cirujanos se matriculan una vez finalizado el pregrado. Esta vía (o su equivalente), que es empleada por la mayoría de las universidades del mundo, se ha mostrado muy poco eficiente para la formación de médicos investigadores. Es preciso que las Facultades de Medicina chilenas consideren que el modelo de programas $\mathrm{MD} / \mathrm{PhD}$ intercalados ofrece los objetivos y una estructura apropiada para potenciar la formación de investigadores clínicos y fortalecer los actuales programas de doctorado. Las acreditaciones de escuelas y programas de doctorado ofrecen la oportunidad para innovar.

Avala esta propuesta las evidencias que muestran que la motivación de los estudiantes puede ser modificada según sean las condiciones del medio educativo y las características personales del estudiante ${ }^{45,46}$. La detección temprana de aptitudes y habilidades, el clima del entorno educativo, el apoyo que recibe el estudiante y el estímulo del desarrollo personal autónomo, facilitan la incorporación de estos a la investigación biomédica. Estas evidencias tienen una significación positiva: un programa de intervención curricular bien diseñado facilitará la opción de los graduados ${ }^{46}$. La mayoría si no todas las escuelas conocen de promisorios jóvenes estudiantes que demuestran una fuerte inquietud por la investigación científica, las sociedades científicas estudiantiles han generado instancias de investigación y publicación pero los estudiantes otorgan una deficiente evaluación a los actuales entrenamientos en investigación ${ }^{47,48}$. En nuestro país, la admisión de postulantes ha sido tradicionalmente vía PSU, la que no emplea criterios de admisión que correlacionen con habilidades o destrezas científicas del postulante, y no se recurre a cursos sistemáticos de nivelación o preparatorios en investigación científica en particular a la entrada de los alumnos, como ocurre en otros países.

Por su proximidad con nuestro contexto cultural y económico, el programa intercalado de la Escuela de Medicina, Universidad Federal de Río de Janeiro, constituye un modelo interesante a homologar, el cual, sin embargo, requiere de un análisis cuidadoso de factores de contexto, organizacionales y presupuestarios.

Les corresponde a las Facultades de Medicina el desafío de progresar hacia una metodología de formación avanzada en investigación que de efectiva solución a la disponibilidad de profesores clínicos que contribuyan a la renovación del conocimiento y que con sus progresos infundan el cuidado de excelencia del paciente.

Agradecimientos: El autor desea agradecer a los directores y responsables de los programas de Doctorados ofrecidos por las Facultades de Medicina Chilenas por la información que proveyeron para este artículo, Drs. Francisco Aboitiz, Doctorado Neurociencias, Pontificia Universidad Católica de Chile; Federico Batiz, Doctorado en Biomedicina, Universidad de los Andes; Josiane Bonnefoy, Doctorado en Salud Pública, Universidad de Chile; Flavio Carrión, Doctorado en Ciencias Médicas, Universidad del Desarrollo; Cecilia Carter, Doctorado en Psicoterapia y Doctorado en Nutrición y Alimentos, Universidad de Chile; Mariano Del Sol, Doctorado en Ciencias Morfológicas, Universidad de La Frontera; Carlos Manterola, Doctorado en Ciencias Médicas, Universidad de la Frontera; Sergio Mezzano, Doctorado en Ciencias Médicas, Universidad Austral de Chile; Jaime Pereira, Doctorado en Ciencias Médicas, Pontificia Universidad Católica de Chile; Juan Redmond, Doctorado en Estudios Interdisciplinarios sobre Pensamiento, Cultura y Sociedad, Universidad de Valparaíso; María Elena Santolaya, Doctorado Ciencias Médicas y Especialidad, Universidad de Chile; Julio Tapia, Doctorado en Ciencias 
Biomédicas, Universidad de Chile; y Benjamín Vicente, Doctorado en Salud Mental, Universidad de Concepción. A los Drs. Carlos Figueroa, Carola Otth y Alejandra Vidal por la revisión del manuscrito y los comentarios que efectuaron; y a la Srta. Carmen Angulo, por el excelente trabajo de búsqueda bibliográfica realizado, la revisión de páginas web y la preparación del material de consulta a las universidades.

\section{Referencias}

1. Resolución de Acreditación Nº629: Doctorado en Ciencias Médicas, Universidad Austral de Chile, 26 de mayo de 2014. Comisión Nacional de Acreditación CNA-Chile. Disponible en: https://www.cnachile.cl/res/ post/RES-POST-00501-02.pdf [Consultado el 9 de junio de 2017].

2. Comisión Nacional de Acreditación. Formulario Solicitud de Acreditación. Programa de Doctorado en Ciencias Médicas, 2013. Universidad Austral de Chile. 2.3. Requisitos de admisión y sistema de selección. [Aprobado según Decreto Rectoría (DR) No 017, 25 de marzo de 2010]. p. 6.

3. Salas SP, Rigotti A. Médicos-Científicos en Chile: ¿Una especie en extinción? Rev Med Chile 2005; 133 (1): 1218.

4. Concha M, Miranda C, Vergara G, Ibarra H. Intención y desarrollo de competencias en investigación clínica en programas de postítulo de médico especialista en Chile. Rev Med Chile 2012; 140 (3): 326-33.

5. Oliveira RV, Campos PCC, Mourão PAS. An MD-PhD program in Brazil: students' concepts of science and of common sense. Braz J Med Biol Res 2011; 44 (11): 110511.

6. Rosenberg LE. The physician-scientist: an essential - and fragile - link in the medical research chain. J Clin Invest 1999; 103 (12): 1621-6.

7. Wyngaarden JB. The clinical investigator as an endangered species. N Engl J Med 1979; 301 (23): 1254-9.

8. Oliveira RV. O Programa de Formação em Pesquisa Médica Md-PhD da UFRJ (tesis de doctorado). Universidade Federal do Rio de Janeiro, Centro de Ciências da Saúde Instituto de Bioquímica Médica, Programa de Educação, Gestão e Difusão em Biociências Doutorado em Química Biológica. Rio de Janeiro, 2009. Capítulo I; p.4-26. Capítulo II; p. 27-33.

9. Park C. Redefining the Doctorate. Discussion Paper. The Higher Education Academy. Innovation Way, York Science Park. Heslington, York, United Kingdom;
January 2007. p. 1-52. Disponible en: https://www.heacademy.ac.uk/system/files/redefining_the_doctorate.pdf [Consultado el 27 de junio de 2017].

10. Altbach PG. Capítulo 1: The Past, Present, and Future of the Research University. En: Altbach PG, Salmi J, Editores, The Road to Academic Excellence. The Making of World-Class Research Universities. Washington DC: The World Bank; 2001. p. 11-32.

11. Brunner JJ. Introducción - La dialéctica público/privado entre el medioevo y la globalización. En: Brunner JJ, Peña C, Editores, El Conflicto de las Universidades: Entre lo Público y lo Privado. Santiago: Ediciones Universidad Diego Portales; 2011. p. 9-61.

12. Norbert Waszek. Philosophie et Geisteswissenschaften à l'Université de Berlin au XIXe siècle. Revue germanique internationale [Internet], 6 | 2007, mis en ligne le 18 octobre 2010, consulté le 30 septembre 2016. Disponible en: http://rgi.revues.org/1063 ; DOI: 10.4000/rgi.1063 [Consultado el 2 de junio de 2017].

13. Wilhelm Von Humboldt (1767-1835). En: Prospects: the quarterly review of comparative education Vol. XXIII, no. 3/4. Paris; 1993. p. 613-23. CUNESCO: International Bureau of Education; 2000. Disponible en: http:// www.ibe.unesco.org/sites/default/files/humbolde.PDF [Consultado el 9 de junio de 2017].

14. Ash MG. Bachelor of What, Master of Whom? The Humboldt Myth and Historical Transformations of Higher Education in German-Speaking Europe and the US. Eur J Educ 2006; 41 (2): 245-67.

15. Kehm BM. Editorial: The Future of the Bologna ProcessThe Bologna Process of the Future. Eur J Educ 2010; 45 (4): 529-34.

16. Motova GN. The Bologna Process: 15 Years Later. Russ Educ Soc 2016; 58 (4): 313-33.

17. Reflexiones y Perspectivas de la Educación Superior en América Latina. Informe Final-Proyecto TuningAmerica Latina 2004-2007. Universidad de Deusto y Universidad de Groningen. Ed. Universidad de Deusto. España; 2007.

18. Patricio M, Harden RM. The Bologna Process-A global vision for the future of medical education. Med Teach 2010; 32 (4): 305-15.

19. Ross MT, Nikolić N, Peeraer G, Murt A, Kroiča J, Elcin $\mathrm{M}$, et al. Report of the MEDINE2 Bachelor of Medicine (Bologna First Cycle) Tuning Project. Med Teach 2014; 36 (4): 314-21.

20. The 2009 Framework for Undergraduate Medical Education in the Netherlands. Professor CLA van Herwaarden, Professor RFJM Laan, RRM Leunissen MSc, Editores, Dutch Federation of University Medical Centres. Utrecht, The Netherlands; Octubre, 
2009. p. 1-87. Disponible en : http://www.nfu.nl/img/ pdf/09.4072_Brochure_Raamplan_artsopleiding_-_Framework_for_Undergraduate_2009.pdf [Consultado el 27 de junio de 2017].

21. Kaiser HJ, Kiessling C. Two-cycle curriculum - bachelor-master structure according to the Bologna agreement: the Swiss experience in Basle. GMS J Med Educ 2010; 27 (2): 1-5.

22. Legifrance.gov.fr [Internet]. Paris: Secrétariat général du Gouvernement, République Française. Code de l'Éducation [Version Consolidée au 12 Mai 2017]. Livre VI: L'organisation des enseignements supérieurs. Titre Ier: L'organisation générale des enseignements, Chapitre III : Collation des grades et titres universitaires. Titre III: Les formations de santé, Chapitre II: Les études médicales. Disponible en: https://www.legifrance.gouv.fr/ affichCode.do?cidTexte=LEGITEXT000006071191\&dateTexte $=20170614$ [Consultado el 12 de junio de 2017].

23. The Education System in the Federal Republic of Germany 2013/2014. A description of the responsibilities, structures and developments in education policy for the exchange of information in Europe. Eds. Brigitte Lohmar y Thomas Eckhardt. Documentation and Education Information Service/ German EURYDICE Unit of the Länder in the Secretariat of the Standing Conference of the Ministers of Education and Cultural Affairs of the Länder in the Federal Republic of Germany in cooperation with the German EURYDICE Unit of the Federal Government in the Federal Ministry of Education and Research, (C KMK. Bonn; 2015. p. 147-173. Disponible en: https://www.kmk.org/fileadmin/Dateien/pdf/Eurydice/Bildungswesen-engl-pdfs/dossier_en_ebook.pdf [Consultado el 9 de junio de 2017].

24. Agencia Estatal Boletín Oficial del Estado [Internet]. Madrid: Ministerio de la Presidencia y para las Administraciones Territoriales, Gobierno de España; [junio, 2017]. Boletín Oficial del Estado Núm. 185 Sec. I. I. Disposiciones Generales. Ministerio de Educación. 13317 Real Decreto 1027/2011, de 15 de julio, por el que se establece el Marco Español de Cualificaciones para la Educación Superior. Agosto 2011, p. 87912-17. Disponible en: http://www.boe.es/boe/dias/2011/08/03/ pdfs/BOE-A-2011-13317.pdf [Consultado el 27 de junio de 2017].

25. Universidad Complutense de Madrid, Facultad de Medicina [Internet]. Madrid: Universidad Complutense de Madrid; [mayo 2017]. Grado en Medicina. Características de la titulación, Medicina. Denominación del título: Grado en Medicina. Nivel de MECES: 3. Disponible en: https://medicina.ucm.es/caracteristicas-de-la-titulacion,-medicina [Consultado el 23 de junio de 2017].
26. QAA: UK Quality Code for Higher Education. Part A: Setting and Maintaining Academic Standards. The Frameworks for Higher Education Qualifications of UK Degree-Awarding Bodies. Gloucester; October 2014. p. 15-18. Disponible en: http://www.qaa.ac.uk/en/ Publications/Documents/qualifications-frameworks.pdf [Consultado el 16 de junio de 2017].

27. O'Connor BN. Perspectives on professional doctorate education in the United States. Work Based Learning e-Journal 2011; 2 (1): 44-64.

28. Heun X, Eisenlöffel C, Barann B, Müller-Hilke B. Dr. med. - obsolete? A cross sectional survey to investigate the perception and acceptance of the German medical degree GMS J Med Educ 2014; 31 (3): 10-20.

29. de Weert E, Aamodt, PO, Carlsten TC, Jeliazkova M. Part Three: Five Case Studies on Curriculum Reform. En The Extent and Impact of Higher Education Curricular Reform Across Europe, Center for Higher Education Policy Studies (CHEPS). University of Twente. Enschede, NL; 2007. p. 5-24.

30. Organización de las Naciones Unidas para la Educación, la Ciencia y la Cultura (UNESCO). Instituto de Estadística de la UNESCO, UNESCO-UIS. Clasificación Internacional Normalizada de la Educación CINE 2011. Montréal, Canadá; 2013. p. 6-81. Disponible en: http:// uis.unesco.org/en/topic/international-standard-classification-education-isced [Consultado el 20 de abril de 2017].

31. Glowinski I, Julian C, Onken J, Zimmerman C, Pion G. MSTP Study: The careers and professional activities of graduates of the NIGMS Bethesda (MD): National Institute of General Medical Sciences, 1998. Disponible en: https://publications.nigms.nih.gov/reports/mstpstudy/ [Consultado el 5 de junio de 2017].

32. The Harvard/M.I.T. MD-PhD Program [Internet]. Boston: Harvard Medical School, Harvard University; [2017]. Disponible en: http://www.hms.harvard.edu/ md_phd/ [Consultado el 5 de junio de 2017].

33. School of Clinical Medicine. $\mathrm{MB} / \mathrm{PhD}$-Course Organization [Internet]. Cambridge: School of Clinical Medicine. Cambridge University; [2017]. Disponible en: http:// www.medschl.cam.ac.uk/education/courses/mbphd/ [Consultado el 5 de junio de 2017].

34. Cox TM, Brimicombe J, Wood DF, Peters DK. The Cambridge Bachelor of Medicine (MB)/Doctor of Philosophy (PhD): graduate outcomes of the first $\mathrm{MB} /$ PhD programme in the UK. Clin Med 2012; 12 (6): 530-34.

35. Deanery of Research, $\mathrm{MD} / \mathrm{PhD}$ Programme [Internet]. Heidelberg: Medizinische Fakultät Heidelberg, Ruprecht-Karls-Universität Heidelberg; [2017]. Disponible en: 
http://www.medizinische-fakultaet-hd.uni-heidelberg. de/About-the-Programme.9575.0.html?\&L=en\#c49261 [Consultado el 5 de junio de 2017].

36. Kuehnlea F, Winklerb DT, Meier-Abta PJ. Swiss national MD-PhD program: an outcome analysis. Swiss Med Wkly 2009; 139 (37-38): 540-6.

37. Bonham AC. MD-PhD Training: Looking Back and Looking Forward. Acad Med 2014; 89 (1): 21-3.

38. Jeffe DB, Andriole DA, Wathington HD, Tai RH. Educational outcomes for students enrolled in $\mathrm{MD}-\mathrm{PhD}$ programs at medical school matriculation, 1995-2000: A national cohort study. Acad Med 2014; 89 (1): 84-93.

39. Schwartz P, Gaulton GN. Addressing the needs of basic and clinical research: analysis of graduates of the University of Pennsylvania MD-PhD program. JAMA 1999; 281 (1): 96-9.

40. Maack T. Dato compilado por Olaf S. Andersen y presentado en la Universidade Estadual de Campinas (UNICAMP) por Thomas Maack (tmaak@med.cornell. edu), 2009. (Citado por Oliveira y col, 2009).

41. Garrison HH, Deschamps AM. NIH research funding and early career physician scientists: continuing challenges in the 21 st century. FASEB J 2014; 28 (3): 1049-58.

42. Stewart GW. An MB PhD programme in the UK: the UCL experience. Clin Med 2012; 12 (6): 526-9.
43. Frenk J, Chen L, Bhutta Z, Cohen J, Crisp N, Evans T, et al. Health professionals for a new century: transforming education to strengthen health systems in an interdependent world. Lancet 2010; 376 (9756): 1923-58.

44. Amgad M, Man Kin Tsui M, Liptrott SJ, Shash E. Medical Student Research: An Integrated Mixed-Methods Systematic Review and Meta-Analysis. PLoS ONE 2015; 10(6): e0127470. doi:10.1371/journal.pone.0127470

45. Orsini C, Vivian I. Binnie V, Wilson SL. Determinants and outcomes of motivation in health professions education: a systematic review based on self-determination theory. J Educ Eval Health Prof 2016; 13: 19. http:// dx.doi.org/10.3352/jeehp.2016.13.19.

46. Krupat E, Camargo CA, Strewler GJ, Gordon J, Espinola JA, Fleenor TJ, et al. Factors associated with physicians' choice of a career in research: a retrospective report 15 years after medical school graduation. Adv in Health Sci Educ 2017; 22: 5-15.

47. Clouet-Huerta DE, Correa K. Investigación médica en pregrado: ¿qué está sucediendo en Chile? Rev Med Chile 2014; 142: 1488-90.

48. Mayta-Tristán P, Cartagena-Klein R, Pereyra-Elías R, Portillo A, Rodríguez-Morales A. Apreciación de estudiantes de Medicina latinoamericanos sobre la capacitación universitaria en investigación científica. Rev Med Chile 2013; 141: 716-22. 